\title{
Losing the battle: perceived status loss and contemplated or attempted suicide in older adults
}

\author{
Alexandre Y. Dombrovski ${ }^{\mathrm{a}}$, Elizabeth Aslinger ${ }^{\mathrm{b}}$, Aidan G.C. Wright ${ }^{\mathrm{a}}$, and Katalin Szanto ${ }^{\mathrm{a}}$ \\ aUniversity of Pittsburgh, School of Medicine

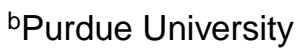

\begin{abstract}
Objective-While loss of socioeconomic status (SES) has been linked to suicidal behavior, it is unclear whether this experience is merely a downstream effect of psychopathology ("downward drift"), a sign of hardship, or an independent psychological contributor to suicide risk. We examined the association between the subjective experience of status loss and suicidal behavior and ideation in old age, while accounting for potential confounders. We were also interested in whether status loss was associated with mere thoughts of suicide vs. suicidal behavior.
\end{abstract}

Methods-Fifty older (55+) depressed suicide attempters, 29 depressed suicide ideators with no history of attempted suicide, 38 non-suicidal depressed participants and 45 non-psychiatric controls underwent detailed clinical characterization and reported their current and highest lifetime SES.

Results-Suicide attempters were more likely to report a decline in their SES compared to healthy controls and non-suicidal depressed older adults, while not differing from suicide ideators. This difference was not explained by objective predictors of SES, including education, financial difficulties, and the presence of addiction. Interestingly, while the current SES of suicide attempters was much lower than that of comparison groups, their reported highest lifetime SES was just as high, despite the differences in education.

Conclusion-In older adults, the experience of status loss is associated with contemplated and attempted suicide even after accounting for objective indicators of social status and psychopathology. It is possible that suicidal individuals retrospectively inflate their previous status, making their current standing appear even worse by comparison.

\section{Keywords}

suicide; socioeconomic status; aged

\section{Introduction}

In the Iliad, Ajax kills himself after losing the competition for Achilles' divine armor to Odysseus. Ajax lost tîmê (roughly corresponding to honor, often taking the form of war

Please address correspondence to: Dr. Alexandre Dombrovski, 100 North Bellefield Ave., Room 742, Pittsburgh, PA 15213, dombax@upmc.edu, (412) 246-6143 (phone), (412) 246-6030 (fax). 
trophies), which translated into social status in the Homeric age. Although the determinants of status have changed, even now its loss is thought to precipitate suicide in the modern West, particularly in white ${ }^{1}$ and older men ${ }^{2,3}$. This may be the case, but empirical knowledge of this phenomenon is limited. Although low socio-economic status (SES) is often linked to suicide, it may simply reflect early-life vulnerability factors such as low education, mental illness, unfavorable family environment and even family history of mental illness ${ }^{4,5}$. At the same time, it does appear that status loss, rather than low SES by itself, is suicidogenic ${ }^{1,6-9}$. What remains uncertain, however, is whether the experience of status loss is merely a downstream effect of psychopathology ("downward drift"), a marker of objective hardship, or an independent contributor to suicide risk.

Further, while status loss may be suicidogenic, one might also wonder why it has a particularly devastating impact on certain individuals. In our work with suicidal patients, we see that such insults are amplified by black-and-white thinking ${ }^{10,11}$. As one's current situation is catastrophized, the past is idealized, creating the sense that one's life has been ruined. This observation informs the prediction that suicidal individuals would perceive their current social status as very low - not without a reality basis - and that they would place themselves considerably higher in their earlier life. The degree of perceived decline in this framework would scale with indices of cognitive distortions such as neuroticism and proneness to regret, both implicated in suicidal behavior or ideation ${ }^{12-15}$. Regret proneness is stronger among people who tend to compare their situation to what might have been if they had made better decisions ${ }^{16,17}$. Such 'if only' thinking is associated with self-blame ${ }^{18}$. Older adults prone to regret often feel that there is no time left for any missteps to be corrected $^{19,20}$ and are more likely to suffer from depression and suicidal ideation ${ }^{21}$.

Motivated by the high suicide rates in late life and the social theory of status-driven suicide in older men, we focused our investigation on older men and women (aged 55+). To separate the suicidogenic effects of status loss from those of psychopathology, participants underwent a detailed psychopathological characterization, and a non-suicidal depressed group was included. Another question yet unanswered in the literature is whether the experience of status loss elicits thoughts of suicide or compels one to act on those thoughts. To address this question, we included a group of depressed older adults with clinically significant suicidal ideation but no lifetime history of attempted suicide. We examined the association between the subjective experience of status loss, suicidal behavior and ideation, and proneness to cognitive distortions, and we hypothesized that attempted suicide will be associated with perceived loss of social status. Second, we explored the psychological correlates of subjective status loss.

\section{Methods}

\section{Sample and its clinical characterization}

All procedures were in accordance with the ethical standards of the University of Pittsburgh IRB (Protocol IRB0407166), and with the Helsinki Declaration. The participants were 162 adults aged 55-85 (mean: 66.5). Participants were separated into groups of suicide attempters with depression $(n=50)$, suicide ideators with depression $(n=29)$, non-suicidal depressed controls $(n=38)$, and healthy controls $(n=49)$. 
The sample was recruited at a psychogeriatric inpatient unit, late-life depression clinic, primary care, and through community advertisements as part of a larger study of suicidal behavior in late life ${ }^{22,23}$. Suicide attempters had a history of a self-injurious act with the intent to die within a 2-week period of completing the study assessments or had a history of a past suicide attempt with strong current suicidal ideation at the time of study enrollment. Suicide attempt history was verified by a psychiatrist (AYD or KSz), using all available information: participant's report, medical records, information from the treatment team, and collateral information from family or friends. Significant discrepancies between these sources precluded participation. Medical seriousness of attempts was assessed using the Beck Lethality Scale (BLS) ${ }^{24}$; for participants with multiple attempts, data for the highestlethality attempt are presented. Twenty-two suicide attempters made a high-lethality attempt (BLS score 24 ) and 28 attempters made a low-lethality attempt (BLS score < 4). Suicidal intent associated with suicide attempts was assessed using Beck's Suicide Intent Scale (SIS). Suicide ideators had suicidal ideation with a specific plan but no lifetime history of suicide attempt. These participants have seriously contemplated suicide and communicated this intention to their family or medical professionals. Participants with passive death wish or transient or ambiguous suicidal ideas were excluded from this group. Non-suicidal depressed older adults were included in the study as a comparison group in order to identify an association between suicidal thoughts or behavior and status loss above and beyond the effects of depression. These participants had a SCID/DSM-IV diagnosis of major depression and a score of 14 or higher on the 17-item Hamilton Rating Scale for Depression (HRSD-17). Non-suicidal depressed participants had no lifetime history of self-injurious behavior, suicidal ideation, or suicide attempts, based on review of medical records, SCID/ DSM-IV, and a score of 0 on the HRSD-17 suicide item. Non-psychiatric controls had no lifetime history of psychiatric disorders, as determined by the SCID/DSM-IV.

\section{Measures}

Participants' subjective (perceived) social status was measured using the MacArthur Sociodemographic Questionnaire ${ }^{25}$. Respondents rated their status using the Scale of Subjective Status (Adler et al., 2000; Ostrove et al., 2000). Participants were instructed:

"Think of this ladder as showing where people stand in their communities. People define community in different ways. Please define it in whatever way is most meaningful to you. At the top of the ladder are the people who have the highest standing in their community. At the bottom are the people who have the lowest standing in their community. Where would you place yourself on this ladder? Place an X on the rung where you think you stand at this time of your life relative to other people in your community."

The ladder has 10 rungs, coded here from 1 at the top to 10 at the bottom. This SES measure has demonstrated adequate test-retest reliability $(\rho=0.62, p<0.01)$ and has been found to predict self-reported health outcomes (Operario et al. 2004). To assess perceived change in social status, we added the following item:

"Think about the time in your life when you were ranked highest on the ladder in comparison to your community. How many steps above your current ranking would you rate yourself? If it is the same now, write " 0 " steps. If it is higher, write the number of steps." 
A text description was also elicited: "When was the time you were at your highest social standing?"

We also assessed psychological characteristics that could contribute to the experience of status loss. Regret proneness. We administered an established 5-item measure developed by Schwartz and colleagues (e.g., "When I think about how I'm doing in life, I often assess opportunities I have passed up") which in non-clinical samples has been associated regret proneness $^{26}$. The response scale ranged from 1 (=completely disagree) to 5 (=completely agree). Internal consistency (a) across items was .66. Neuroticism. Big Five personality dimensions (neuroticism, extraversion, openness, agreeableness and conscientiousness) were assessed using the NEO Five-Factor Inventory (NEO) ${ }^{27}$. Neuroticism reflects anxiety, hostility, depression, impulsiveness and vulnerability. Premorbid verbal intelligence was assessed with the Wechsler Test of Adult Reading 28 .

\section{Data Processing and Quality Checks}

A research assistant and a study psychiatrist (AYD) inspected all SES decline ratings vs. the current SES ratings and text descriptions and identified 11 cases where the highest SES would have fallen outside of the range. Since such a drop was numerically implausible, we took the conservative approach of recoding these values such that the highest SES took the minimum possible value (highest rank $=1$ ). In one additional case the reported SES decline (2 steps) appeared to contradict the text description ("When I was taking care of my 6 kids"). This case was marked as unclear. The recoded and unclear cases were excluded in sensitivity analyses. Furthermore, to rule out that our results are driven by participants' errors in the scaling of the SES decline, we performed sensitivity analyses described below.

Outlier analyses were conducted to detect scores which fell beyond 3 standard deviations from the models' predictions. No outliers were found. One participant was missing the years of education variable, nine $(6 \%)$ were missing the WTAR score, and $11(7 \%)$ were missing the regret subscale score, all of which were imputed. Forty-seven participants (29\%) were missing NEO-FFI scores, and list-wise deletion was used in the main analysis using neuroticism as a predictor. These data were assumed to be missing at random because the NEO-FFI was introduced late in the study. To ensure that using the full data did not result in diverging conclusions, we also imputed the NEO-FFI values using multiple imputation by chained equations (R package mice) and conducted a sensitivity analysis on 22 imputed datasets $^{29}$.

\section{Data analyses}

All analyses were performed in $\mathrm{R}$, version 3.3.2. We aimed to examine group differences between suicide attempters, suicide ideators, and the two comparison groups (non-suicidal depressed and healthy controls) in the social status drop, while incorporating the intercept in their social status. We controlled for income and education across all models in order to examine differences related specifically to suicidal behavior beyond objective determinants of social status, as there appeared to be group differences on these variables (Table 1). Toward this end, we first calculated the subjects' highest reported social status by adding their self-reported drop to their self-reported current status. Taking advantage of two 
observations per person, we then set up a linear mixed-effects (LME) model predicting social status at both time-points from group membership and co-variates (Ime4 and ImerTest R packages; model-predicted means: lsmeans). A significant group*time interaction would correspond to our first hypothesis of group differences in status loss. We then tested whether psychological characteristics similarly interacted with time.

Since participants rate the SES decline retrospectively, it could be argued that its scaling is somehow different in people who currently find themselves in different parts of the MacArthur ladder. To rule out the possibility that our findings were entirely explained by such scaling differences, we conducted a number of sensitivity analyses. First, we examined predictors of SES decline after co-varying for current SES. We note that this amounts to over-controlling, because current SES is a result of any preceding drop (subjective or objective), and such an analysis inevitably under-estimates effects of predictors. The SES decline variable had a non-normal distribution and a large mass of zeros. Its variance (4.463) was quite large relative to the mean (2.096), which would indicate over-dispersion from a Poisson distribution. Therefore, we regressed SES decline on current status, group and other predictors using a negative binomial multiple regression $(\mathrm{g} / \mathrm{m} . \mathrm{nb})$ to analyze the variance accounted for by group differences, which was not accounted for by current standing. Second, we examined whether suicide attempters were more likely to report any SES decline (binary variable) than the comparison groups, using binary logistic regression. Suicide attempters served as a reference group in all analyses. All analyses started with a larger set of explanatory variables, and the best-fitting model was subsequently selected based on the Akaike Information Criterion.

\section{Results}

\section{Group differences in perceived SES decline}

Group characteristics are presented in Table 1. Suicide attempters had a lower level of education; the groups were demographically similar otherwise. The three depressed groups did not differ in the lifetime prevalence of substance use or anxiety disorders.

Our main LME analysis revealed that both suicide attempters and suicide ideators reported a lower overall SES compared to healthy controls and non-suicidal depressed individuals, while the two suicidal groups did not differ from each other (Table 2). Confirming our hypothesis, suicide attempters (SA) reported a greater SES decline than healthy controls (HC) and non-suicidal depressed (DC), not differing from suicide ideators (SI; Table 2; Fig. 1; post-hoc contrast of contrasts: group 1: C, D; group 2: D, SI, group 3: I, SA). These results were unchanged with or without co-varying for objective determinants of SES: age, income, education and addiction diagnosis (any current substance abuse or dependence; effects of lifetime diagnosis were similar, data not shown). Interestingly, addiction (current or lifetime) predicted lower overall SES, but not greater decline. Since suicide ideators and attempters suffered from more severe depression than non-suicidal depressed participants (Table 1), we included HRSD-16 score and its interaction with time as covariates (Table 2, model 5). Greater depression severity predicted overall lower reported SES with no effect on decline; the difference between suicide attempters and non-suicidal depressed in SES decline was unchanged. Race and sex had no significant main effects or interactions with 
time and their deletion from the model improved fits (data not shown). We nevertheless tested whether group differences in SES decline were accentuated in males, and this was not the case $\left(\chi^{2}{ }_{3}=1.34, p=0.72\right)$. Further, we examined whether ostensibly objective SES determinants such as not being able to make ends meet, changes in income, or being in debt (all assessed by MacArthur items) explained group differences. The effect of group on SES change remained essentially unchanged after accounting for all of these factors $\left(\chi^{2}{ }_{3}=\right.$ 23.37, $p<0.001)$.

A post-hoc analysis showed that while current SES was much lower in suicide attempters vs. the comparison groups $\left(\mathrm{F}_{3,158}=17.7, p<0.001\right.$, Tukey pairwise comparisons: $\mathrm{SA}>\mathrm{DC}>\mathrm{HC}$, $\mathrm{SI}>\mathrm{HC})$, their reported highest lifetime SES was just as high $\left(\mathrm{F}_{3,158}=1.6, p>0.18\right)$.

\section{Psychological correlates of perceived SES decline (Table 3)}

We proceeded to examine psychological correlates of status loss in LME models. Because regret-proneness and neuroticism were inter-correlated $\left(r_{115}=0.42\right)$, we first tested each predictor separately, noting that they may tap into the same construct. After accounting for age, income, education and the diagnosis of addiction, regret-proneness $\left(\mathrm{t}_{156}=-3.3, p=.001\right.$; Table 3, model 1) and neuroticism $\left(\mathrm{t}_{109}=-3.3, p=.001\right.$; Table 3, model 2$)$ predicted greater perceived decline in social status. These effects, however, became smaller and nonsignificant once group was included in the model (Table 3, model 4). A post-hoc analysis excluding the healthy controls indicated that this was mostly due to the differences in both neuroticism and SES decline between controls and the three depressed groups (Table 1). Thus, the effects of both neuroticism and regret were confounded with those of depression.

Sensitivity analyses-First, we tested the models including neuroticism as a predictor in 22 datasets where missing neuroticism values $(n=47)$ were imputed to ensure that the results were robust when using all data. The effect of neuroticism on drop in SES was unchanged with $\left(t_{110.39}=-2.3, p=.002\right)$ and without $\left(t_{117.85}=-3.4, p<.001\right)$ co-varying for regret-proneness. However, this effect was still mostly explained by depression.

To rule out the possibility that our findings were explained by differential scaling of SES decline, we examined group differences in the SES decline by itself, using negative binomial regression. After controlling for age, income, education and addiction, SES decline was still greater in suicide attempters, compared to healthy controls $\left(t_{154}=-5.4, p<.001\right)$ and nonsuicidal depressed individuals $\left(t_{154}=-2.8, p=.006\right)$. The difference between suicide attempters and healthy controls $\left(t_{153}=-3.6, p=.001\right)$, but not non-suicidal depressed individuals $\left(\mathrm{t}_{153}=-1.3, p=.18\right)$ persisted even after accounting for current SES, which amounts to over-controlling, since objective or subjective current SES ostensibly results from objective or subjective drop. Suicide attempters did not differ from suicide ideators in either analysis $(|t|<0.71, p>.47)$. Furthermore, suicide attempters were also more likely to report any drop in their SES compared to healthy controls $\left(z_{154}=-3.5, p<.001\right)$ and to nonsuicidal depressed individuals $\left(z_{154}=-2.1, p=.032\right)$ in a binary logistic regression after controlling for age, income, education and current addiction, suggesting that these group differences are robust to the scaling of reported SES decline. Next, we repeated our main LME analysis after excluding 12 participants with questionable SES decline values. The 
results were again qualitatively unchanged: suicide attempters reported a greater SES decline, compared to healthy controls $\left(t_{142}=4.7, \mathrm{p}<.001\right)$ and non-suicidal depressed individuals $\left(t_{142}=3.1, \mathrm{p}=.002\right)$, but not to suicide ideators $\left(t_{142}=0.3, p=.74\right)$.

\section{Discussion}

In this retrospective case-control study, we examined the experience of socio-economic status (SES) loss among depressed older adults with a history of attempted suicide. They were more likely to report a decline in their SES compared to healthy controls and nonsuicidal depressed older adults, while not differing from depressed individuals with serious suicidal ideation but no history of attempts. This difference was not explained by objective predictors of SES, including education, income, change in income, financial difficulties, debt, and the presence of addiction. Interestingly, while the current SES of suicide attempters was much lower than that of comparison groups, their reported highest lifetime SES was just as high, despite the differences in education. Whereas individuals who reported the greatest status loss were characterized by proneness to regret and neuroticism, these effects were confounded with those of depression. While a retrospective study cannot establish a causal chain of events and experiences that result in contemplating or attempting suicide, these findings add to our understanding of the relationship between subjective social status and suicide. We assume that many individuals with a history of suicide attempt had indeed experienced a decline in their objective social status. However, the fact that group differences in status loss are not explained by objective indicators and the almost implausibly high earlier SES reported by suicide attempters suggest that, in some individuals, highest lifetime SES may have been retrospectively inflated. Below, we discuss our results in terms of existing theories of suicide and address the limitations of our study.

Our study extends earlier findings ${ }^{1,6-9}$, demonstrating a robust association between perceived status loss and attempted or contemplated suicide in depressed older adults. Further, our study rules out several possible confounders of this relationship, including practical hardship (debt, financial difficulties, changes in income or low income). It also does not appear from our data that the experience of status loss in suicidal individuals is merely a downstream effect of psychopathology, although both addiction and depression/ neuroticism were among significant independent predictors. Finally, as with most known risk factors ${ }^{30}$, perceived status loss was just as prevalent in suicide ideators as in suicide attempters, possibly suggesting that it operates at the stage of the emergence of suicidal ideas and does not necessarily catalyze their implementation. This negative result, however, should be taken with caution, since some attempts in this sample were remote, and a group comprised entirely of recent attempters could have differed from ideators.

The question of dispositional vulnerability to status loss remains unanswered. While individual differences in neuroticism and regret-proneness were predictive of self-reported status loss, these effects were explained by the presence of depression. This is not surprising in light of neuroticism's broad and non-specific association with many forms of dysfunction, including stressful life events, as well as depression and virtually all mental disorders ${ }^{31,32}$. Further consideration of personality processes is needed to understand what contributes to the heightened experience of status loss in suicidal individuals. A more detailed 
investigation of cognitive distortions would constitute a better test of Neuringer's dichotomous thinking hypothesis in this context ${ }^{11}$. Another candidate personality feature is pathological narcissism. While lay and DSM conceptions of narcissism emphasize grandiosity, the clinical literature also recognizes narcissistic vulnerability, in the form of contingent self-esteem, intense feelings of shame, and interpersonal and affective dysregulation ${ }^{33}$. Although often studied using separate dispositional tendencies, narcissistic grandiosity and vulnerability can be thought of as different states pathologically narcissistic individuals oscillate between ${ }^{34}$, potentially paralleling extremes of perceived social status. Of particular interest in this context is our finding of relatively high previous social status reported by suicidal individuals. Pathological narcissism has been found to associated with attempted suicide ${ }^{35}$ and its recurrence (even when controlling for borderline features ${ }^{36}$ ), although this area has been relatively neglected since the early psychoanalytic work on narcissistic collapse and suicide ${ }^{37,38}$.

Some limitations qualify our findings. First, unobserved confounders are always a potential problem in case-control studies and status loss in suicidal elderly may be epiphenomenal to an unmeasured confounder, although we have taken care to rule out the likely candidates such as poverty, loss of income, debt, general financial difficulties and various forms of psychopathology including addiction. Second, our findings may not generalize to people who die by suicide, although the inclusion of many high-lethality attempters partly mitigates this concern. Next, the retrospective assessment of highest lifetime status makes it difficult to understand to what extent suicidal individuals actually perceived their status as high in the past, and we cannot definitively claim that the latter is inflated retrospectively. Further, given that some suicide attempts were remote in some participants, we cannot rule out the possibility that these attempts predated the drop in SES. Finally, our findings may not generalize to suicidal individuals without major depression.

In conclusion, we found that, in older adults with depression, the experience of a decline in socio-economic status (SES) was related to attempting and contemplating suicide. This relationship was not explained by objective indicators of social status or by psychopathology and addiction. It is possible, on the other hand, that suicidal individuals retrospectively inflate their previous status, making their current standing appear even worse. Further research is needed on how suicidal individuals appraise the experience of status loss and what vulnerability factors render challenges to one's social status suicidogenic.

\section{Acknowledgments}

Funding: This work was funded by the National Institutes of Health, Bethesda, Maryland, USA (MH R01 MH100095 and MH048463 to A.D.; MH R01MH085651 to K.Sz.; and UL1 TR001857 and L30 MH101760 to AGW.) The funding agency had no role in the design and conduct of the study; the collection, management, analysis, and interpretation of the data; preparation, review, or approval of the manuscript; or decision to submit the manuscript for publication.

\section{References}

1. Breed W. Occupational Mobility and Suicide Among White Males. Am Sociol Rev. 1963; 28(2): 179-188.

2. Canetto SS. Gender and suicide in the elderly. Suicide Life Threat Behav. 1992; 22(1):80-97. [PubMed: 1579988] 
3. Canetto SS, Sakinofsky I. The Gender Paradox in Suicide. Suicide Life Threat Behav. 1998; 28(1): 1-23. [PubMed: 9560163]

4. Agerbo E, Nordentoft M, Mortensen PB. Familial, psychiatric, and socioeconomic risk factors for suicide in young people: nested case-control study. BMJ. 2002; 325(7355):74. [PubMed: 12114236]

5. Lorant V, Kunst AE, Huisman M, et al. Socio-economic inequalities in suicide: a European comparative study. Br J Psychiatry. 2005; 187(1):49-54. [PubMed: 15994571]

6. Coope C, Donovan J, Wilson C, et al. Characteristics of people dying by suicide after job loss, financial difficulties and other economic stressors during a period of recession (2010-2011): A review of coroners' records. J Affect Disord. 2015:18398-105.

7. Lewis G, Sloggett A. Suicide, deprivation, and unemployment: record linkage study. BMJ. 1998; 317(7168):1283-1286. [PubMed: 9804714]

8. Maris R. Suicide, status, and mobility in Chicago. Soc Forces. 1967; 46(2):246-256.

9. Schneider B, Grebner K, Schnabel A, et al. Impact of employment status and work-related factors on risk of completed suicide. Psychiatry Res. 2011; 190(2):265-270. [PubMed: 21890214]

10. Beck, AT. Cognitive Therapy of Depression. Guilford Press; 1979. p. 442

11. Neuringer C. Dichotomous evaluations in suicidal individuals. J Consult Psychol. 1961; 25(5):445. [PubMed: 14479241]

12. Beautrais AL, Joyce PR, Mulder RT. Personality Traits and Cognitive Styles as Risk Factors for Serious Suicide Attempts among Young People. Suicide Life Threat Behav. 1999; 29(1):37-47. [PubMed: 10322619]

13. Bruine de Bruin W, Dombrovski AY, Parker AM, et al. Late-life Depression, Suicidal Ideation, and Attempted Suicide: The Role of Individual Differences in Maximizing, Regret, and Negative Decision Outcomes. J Behav Decis Mak. 2015:n/a-n/a.

14. Useda JD, Duberstein PR, Conner KR, et al. Personality differences in attempted suicide versus suicide in adults 50 years of age or older. J Consult Clin Psychol. 2007; 75(1):126-33. [PubMed: 17295571]

15. Wiktorsson S, Berg AI, Billstedt E, et al. Neuroticism and extroversion in suicide attempters aged 75 and above and a general population comparison group. Aging Ment Health. 2013; 17(4):479488. [PubMed: 23336286]

16. Delacroix E, Jourdan P. Consumer Tendency to Regret: Validation of a Measurement Scale. Rech Appl En Mark Engl Ed. 2007; 22(1):25-43.

17. Schwartz B, Ward A, Monterosso J, et al. Maximizing versus satisficing: Happiness is a matter of choice. J Pers Soc Psychol. 2002; 83(5):1178-1197. [PubMed: 12416921]

18. Davis CG, Nolen-Hoeksema S, Larson J. Making sense of loss and benefiting from the experience: two construals of meaning. J Soc Psychol. 1998; 75(2):561-74.

19. Västfjäll, D., Peters, E., Bjälkebring, P. Emotion Regulation and Well-Being. Springer; New York, NY: The Experience and Regulation of Regret Across the Adult Life Span; p. 165-180.

20. Wrosch C, Schulz R, Heckhausen J. Health stresses and depressive symptomatology in the elderly: the importance of health engagement control strategies. Health Psychol. 2002; 21(4):340-8. [PubMed: 12090676]

21. de Bruin WB, Dombrovski AY, Parker AM, et al. Late-life Depression, Suicidal Ideation, and Attempted Suicide: The Role of Individual Differences in Maximizing, Regret, and Negative Decision Outcomes. J Behav Decis Mak. 2016; 29(4):363-371. [PubMed: 27840559]

22. Gujral, S., Ogbagaber, S., Dombrovski, AY., et al. Course of cognitive impairment following attempted suicide in older adults. Int J Geriatr Psychiatry. 2015. http://onlinelibrary.wiley.com/doi/ 10.1002/gps.4365/pdfaccessed July 26, 2016

23. Szanto K, de Bruin WB, Parker AM, et al. Decision-making competence and attempted suicide. J Clin Psychiatry. 2015; 76(12):1590-1597.

24. Beck AT, Beck R, Kovacs M. Classification of suicidal behaviors: I. Quantifying intent and medical lethality. Am J Psychiatry. 1975; 132(3):285-7. [PubMed: 1115273]

25. Adler NE, Epel ES, Castellazzo G, et al. Relationship of subjective and objective social status with psychological and physiological functioning: preliminary data in healthy white women. Health Psychol Off J Div Health Psychol Am Psychol Assoc. 2000; 19(6):586-592. 
26. Parker A, Bruine de Bruin W, Fischhoff B. Maximizers versus satisficers: Decision-making styles, competence, and outcomes. Judgement Decis Mak. 2007; 2(6):342-350.

27. Costa, PT., McCrae, RR. The NEO-PI/NEO-FFI manual supplement. Odessa, FL: Psychological Assessment Resources; 1989.

28. Wechsler, D. The Wechsler Test of Adult Reading: WTAR. San Antonio: Psychological Corporation; 2001.

29. White IR, Royston P, Wood AM. Multiple imputation using chained equations: Issues and guidance for practice. Stat Med. 2011; 30(4):377-399. [PubMed: 21225900]

30. May AM, Klonsky ED. What Distinguishes Suicide Attempters From Suicide Ideators? A MetaAnalysis of Potential Factors. Clin Psychol Sci Pract. 2016; 23(1):5-20.

31. Kotov, R., Gamez, W., Schmidt, F., et al. Linking "big” personality traits to anxiety, depressive, and substance use disorders: A meta-analysis. American Psychological Association. 2010. http:// psycnet.apa.org/journals/bul/136/5/768/accessed September 19, 2017

32. Liu RT, Alloy LB. Stress generation in depression: A systematic review of the empirical literature and recommendations for future study. Clin Psychol Rev. 2010; 30(5):582-593. [PubMed: 20478648]

33. Cain NM, Pincus AL, Ansell EB. Narcissism at the crossroads: Phenotypic description of pathological narcissism across clinical theory, social/personality psychology, and psychiatric diagnosis. Clin Psychol Rev. 2008; 28(4):638-656. [PubMed: 18029072]

34. Pincus AL, Cain NM, Wright AG. Narcissistic grandiosity and narcissistic vulnerability in psychotherapy. Personal Disord Theory Res Treat. 2014; 5(4):439-443.

35. Pincus AL, Ansell EB, Pimentel CA, et al. Initial construction and validation of the Pathological Narcissism Inventory. Psychol Assess. 2009; 21(3):365. [PubMed: 19719348]

36. Ansell EB, Wright AGC, Markowitz JC, et al. Personality Disorder Risk Factors for Suicide Attempts over 10 Years of Follow-up. Personal Disord. 2015; 6(2):161-167. [PubMed: 25705977]

37. Maltsberger JT. The descent into suicide. Int J Psychoanal. 2004; 85(3):653-668. [PubMed: 15228702]

38. Ronningstam EF, Maltsberger JT. Pathological narcissism and sudden suicide-related collapse. Suicide Life Threat Behav. 1998; 28(3):261-271. [PubMed: 9807772] 


\section{Key Points}

- In older adults, the experience of socioeconomic status loss is uniquely related to contemplated and attempted suicide.

- $\quad$ This relationship persists after accounting for objective indicators of social status and psychopathology.

- $\quad$ It is possible that suicidal individuals retrospectively inflate their previous status, making their current standing appear even worse by comparison. 


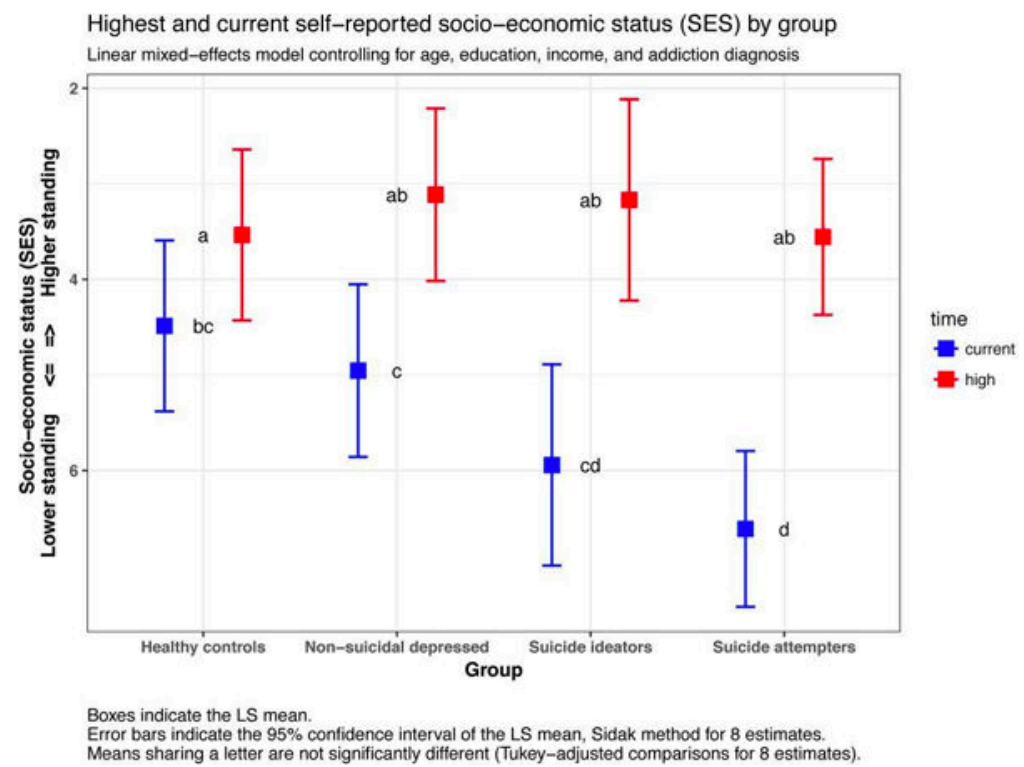

Fig. 1.

Linear mixed-effects model controlling for age, education, income, and addiction diagnosis. 


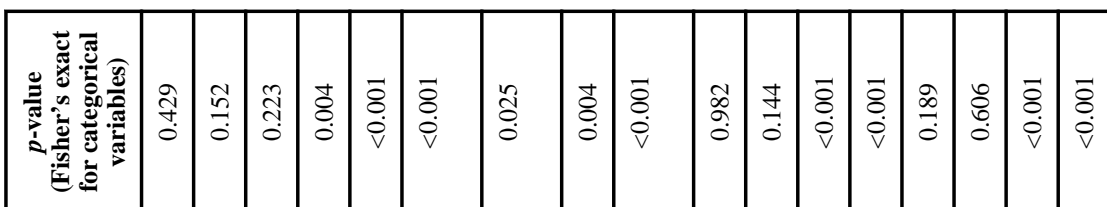

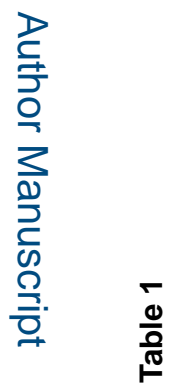

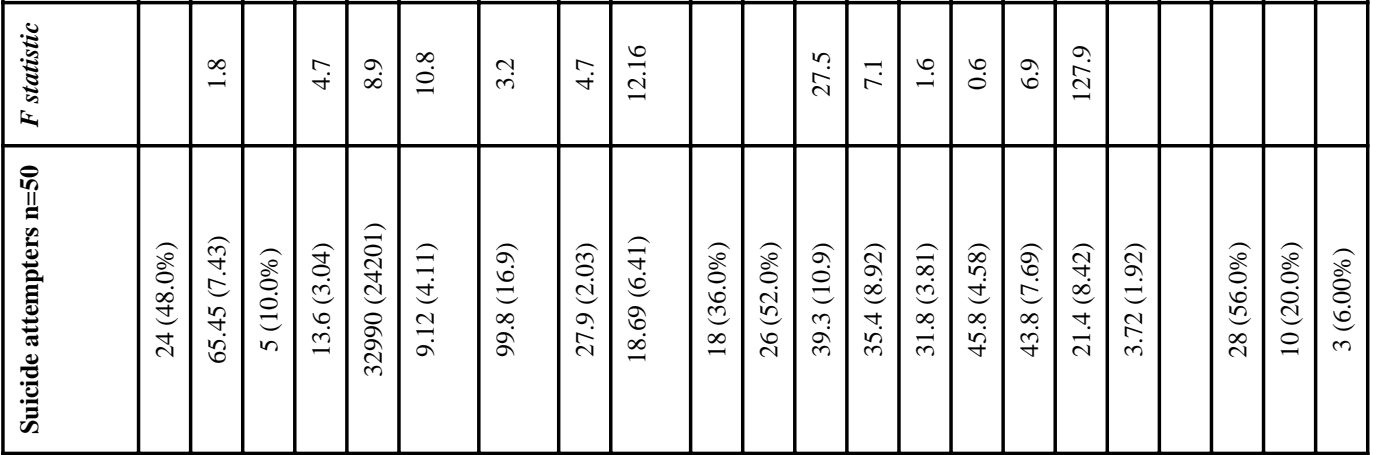

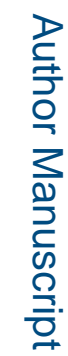

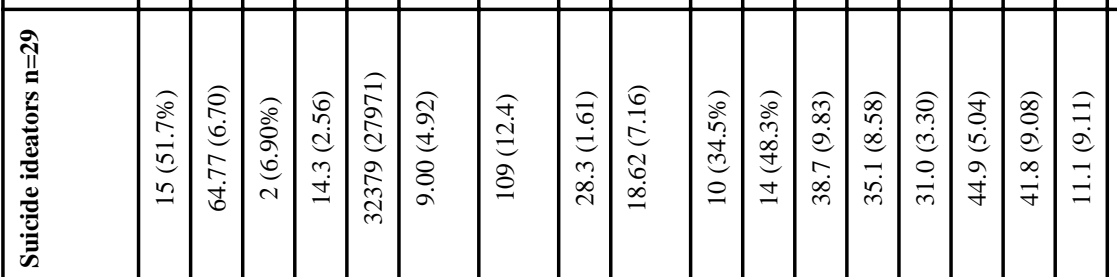

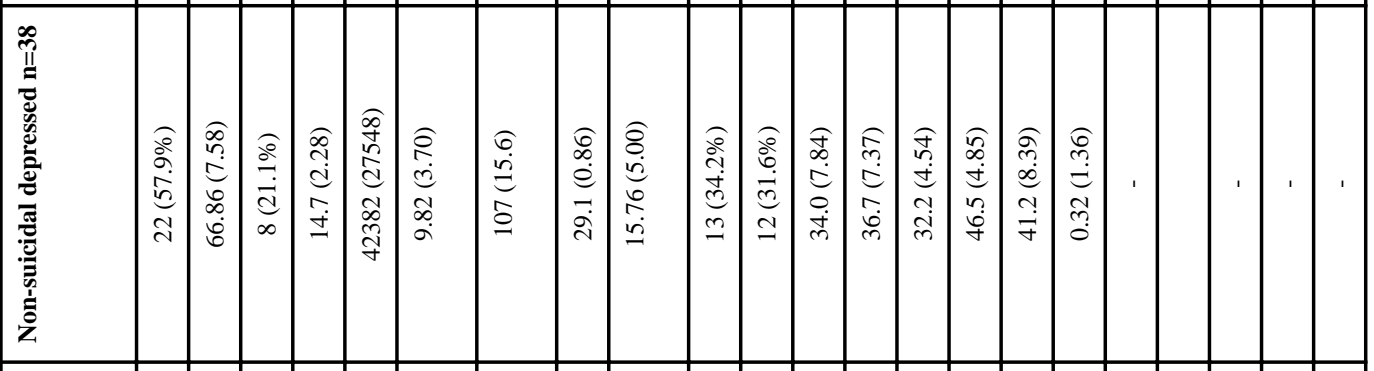

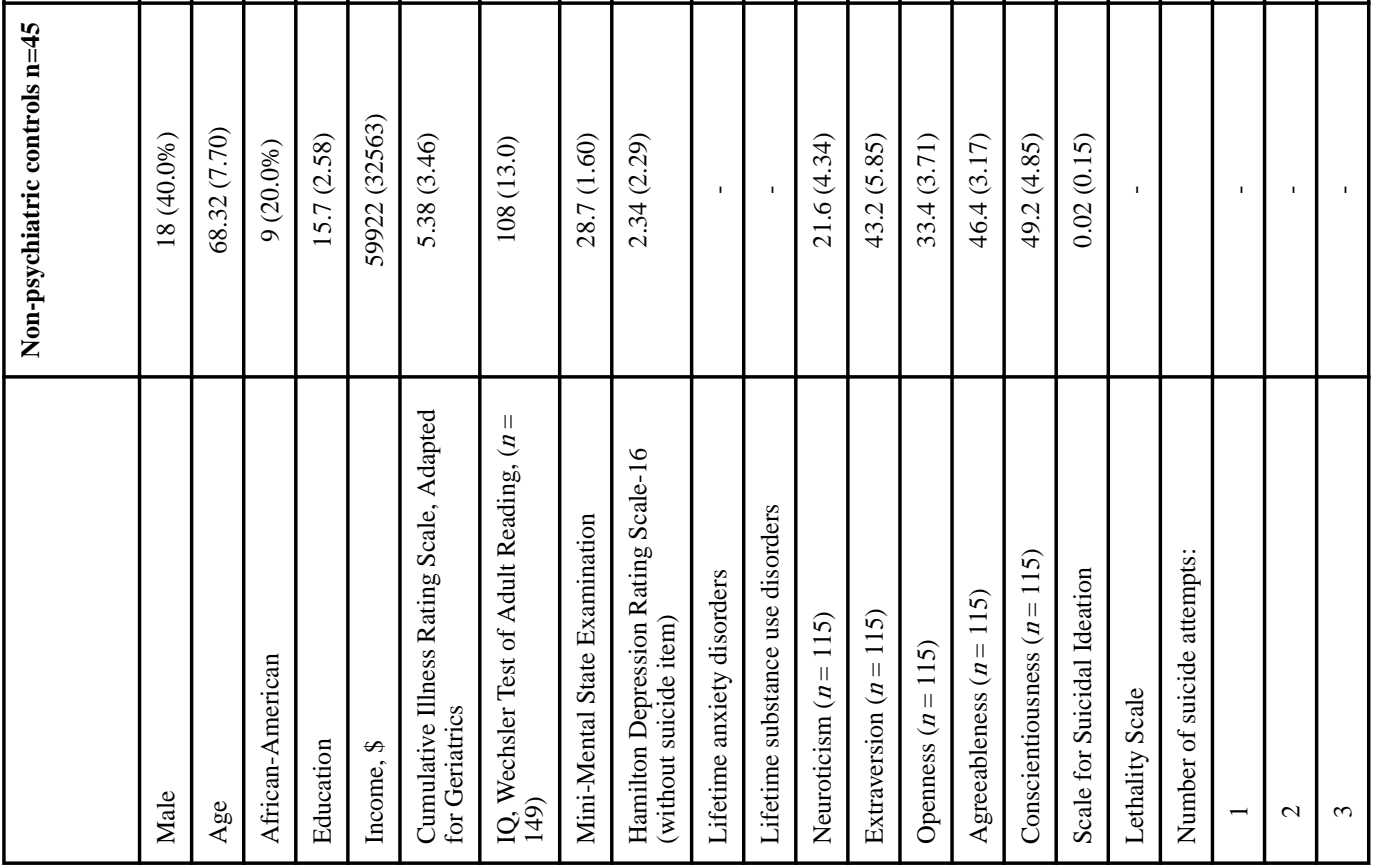

Int J Geriatr Psychiatry. Author manuscript; available in PMC 2019 July 01. 


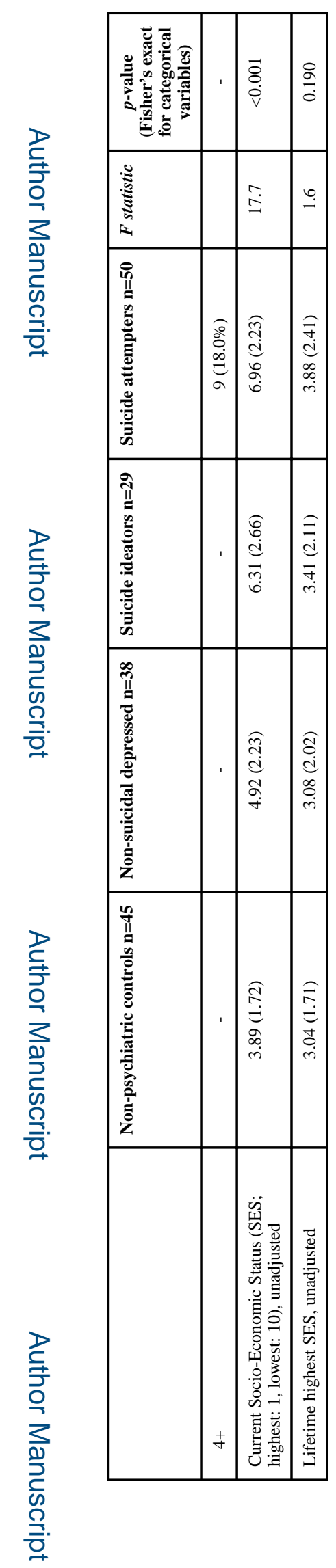

Int J Geriatr Psychiatry. Author manuscript; available in PMC 2019 July 01. 


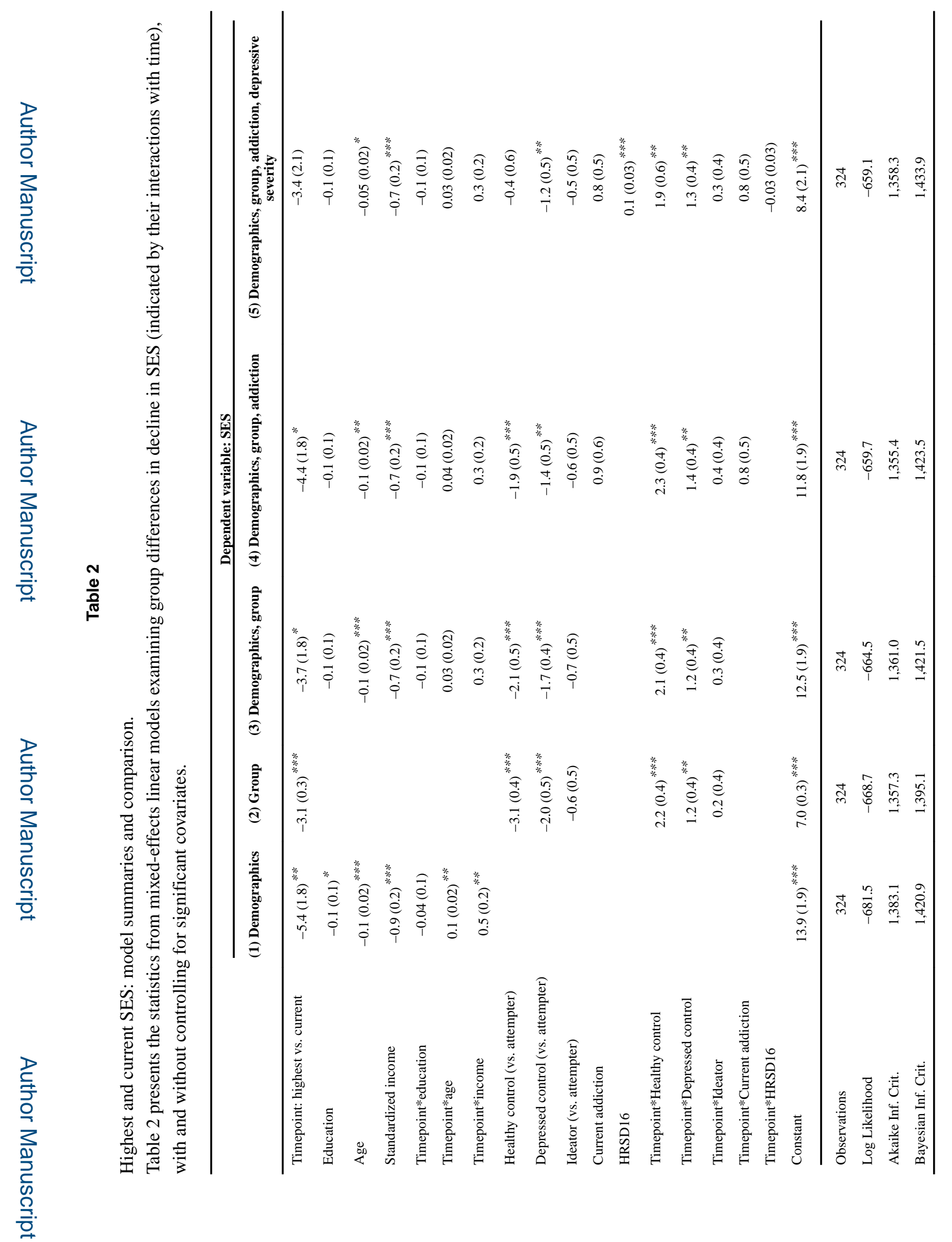

Int J Geriatr Psychiatry. Author manuscript; available in PMC 2019 July 01. 

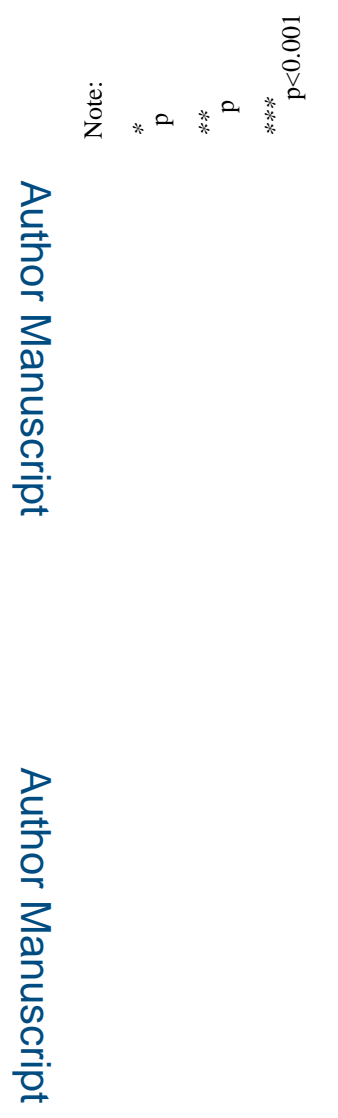

로을

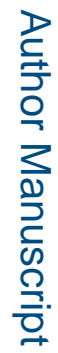

Int J Geriatr Psychiatry. Author manuscript; available in PMC 2019 July 01. 


\section{Table 3}

Psychological correlates of SES decline

Table 3 presents the statistics from mixed-effects linear models examining psychological predictors of decline in SES (indicated by their interactions with time), after controlling for significant covariates.

\begin{tabular}{|c|c|c|c|c|}
\hline & \multicolumn{4}{|c|}{ Dependent variable: SES } \\
\hline & $\begin{array}{c}(1) \\
\text { Demographics, addiction, regret }\end{array}$ & $\begin{array}{l}(2) \\
\text { Demographics, addiction, neuroticism }\end{array}$ & $\begin{array}{c}\text { (3) } \\
\text { Demographics, group, addiction }\end{array}$ & $\begin{array}{c}\text { (4) } \\
\text { Demographics, } \\
\text { group, } \\
\text { addiction, } \\
\text { neuroticism, } \\
\text { regret }\end{array}$ \\
\hline Education & $-0.03(0.08)$ & $-0.01(0.08)$ & $-0.06(0.08)$ & $-0.01(0.08)$ \\
\hline $\begin{array}{l}\text { Timepoint: highest vs. } \\
\text { current }\end{array}$ & $-1.80(2.61)$ & $-0.97(2.55)$ & $-4.42(2.32)$ & $-2.75(2.69)$ \\
\hline Age & $-0.06(0.03)^{*}$ & $-0.04(0.03)$ & $-0.05(0.03)$ & $-0.04(0.03)$ \\
\hline Standardized income & $-0.99(0.21)^{* * *}$ & $-0.92(0.20)^{* * *}$ & $-0.81(0.22)^{* * *}$ & $-0.88(0.22)^{* * *}$ \\
\hline Regret-proneness & $0.17(0.05)^{* *}$ & & & $0.08(0.06)$ \\
\hline Neuroticism & & $0.08(0.02)^{* * * *}$ & & $0.06(0.02)^{* *}$ \\
\hline Current addiction & & & $-1.89(0.55)^{* * *}$ & $-0.44(0.69)$ \\
\hline Control (vs. attempter) & & & $-1.39(0.52)^{* *}$ & $-0.89(0.53)$ \\
\hline $\begin{array}{l}\text { Non-suicidal } \\
\text { depressed (vs. } \\
\text { attempter) }\end{array}$ & & & $-0.61(0.57)$ & $-0.51(0.55)$ \\
\hline Ideator (vs. attempter) & $1.60(0.63)^{*}$ & $1.98(0.60)^{* *}$ & $1.11(0.67)$ & $1.39(0.68)^{*}$ \\
\hline Timepoint*education & $-0.08(0.08)$ & $-0.09(0.08)$ & $-0.06(0.08)$ & $-0.08(0.08)$ \\
\hline Timepoint*age & $0.04(0.03)$ & $0.03(0.03)$ & $0.03(0.03)$ & $0.03(0.03)$ \\
\hline Timepoint*income & $0.49(0.21)^{*}$ & $0.45(0.21)^{*}$ & $0.21(0.21)$ & $0.22(0.22)$ \\
\hline Timepoint*regret & $-0.13(0.06)^{*}$ & & & $-0.06(0.06)$ \\
\hline Timepoint*neuroticism & & $-0.06(0.02)^{* * *}$ & & $-0.01(0.02)$ \\
\hline Timepoint*Control & & & $2.36(0.54)^{* * *}$ & $1.95(0.69)^{* *}$ \\
\hline Timepoint*Depressed & & & $1.56(0.51)^{* *}$ & $1.42(0.53)^{* *}$ \\
\hline Timepoint*Ideator & & & $0.46(0.55)$ & $0.44(0.55)$ \\
\hline Timepoint*addiction & $0.32(0.65)$ & $0.02(0.62)$ & $1.06(0.65)$ & $1.07(0.68)$ \\
\hline Constant & $7.00(2.54)^{* * *}$ & $5.70(2.48)^{*}$ & $10.26(2.39)^{* * * *}$ & $5.48(2.68)^{*}$ \\
\hline Observations & 230 & 230 & 230 & 230 \\
\hline Log Likelihood & -474.95 & -471.62 & -463.66 & -467.08 \\
\hline Akaike Inf. Crit. & 977.90 & 971.25 & 963.33 & 978.16 \\
\hline Bayesian Inf. Crit. & $1,026.04$ & $1,019.38$ & $1,025.21$ & $1,053.80$ \\
\hline \multicolumn{5}{|l|}{ Note: } \\
\hline \multicolumn{5}{|l|}{$*$ p $<0.05$} \\
\hline \multicolumn{5}{|l|}{ *** $\mathrm{p}<0.01$} \\
\hline $\mathrm{p}<0.001$ & & & & \\
\hline
\end{tabular}

